\title{
Parent Preferences for Ulnar Polydactyly Management
}

\author{
Gunnar Goebel ${ }^{1}$, Scott Loewenstein ${ }^{2}$, Joshua Adkinson ${ }^{2}$ \\ ${ }^{1}$ Indiana University School of Medicine; ${ }^{2}$ Indiana University School of Medicine, Department of \\ Plastic Surgery
}

Introduction: Type B ulnar polydactyly is one of the most commonly encountered congenital hand differences and can be treated with either suture ligation or surgical excision. The purpose of this study was to determine what factors families consider in selecting treatment for their child with type B ulnar polydactyly.

Methods: We developed an ad-hoc survey instrument for parents of children with type B ulnar polydactyly that assessed motivation for choosing treatment, parent-reported outcomes, and overall satisfaction. Face validity was confirmed with a think-out-loud protocol using 5 test subjects. We administered surveys via telephone after treatment was complete. We assessed for differences between the cohort who chose in-office suture ligation versus the cohort who opted for operating-room excision using Chi square and Fischer exact tests for categorical variables and Student t-test for continuous variables.

Results: Seventy of the 156 parents of consecutive patients contacted agreed to participate (45\% response rate), with a mean follow-up of 2.25 years. Twenty-eight chose in-office suture ligation and 42 chose surgical excision. Rapid treatment was prioritized more often in those who selected suture ligation than in those who opted for surgical excision $(p=0.044)$. The complication rate for suture ligation was significantly higher than for surgical excision $(p<0.0001$ ), with the most common complication being a residual bump (i.e. nubbin or neuroma stump) (64\%). Respondents with residual bumps reported significantly less satisfaction with the aesthetic appearance of their child's hand $(p<0.001)$ and with their child's treatment outcome $(p=0.028)$ compared to those without residual bumps.

Conclusion: Factors considered by parents in choosing type of treatment for type B ulnar polydactyly vary and may be significantly influenced by the surgeon. Time to treatment plays a determinative role in parents opting for suture ligation rather than surgical excision. Regardless of treatment, the majority of parents remain extremely satisfied with the outcomes. 